\title{
Mechanisms of optic disc swelling with diffuse choroidal melanomas: clinicopathologic correlations
}

\author{
GARY C. BROWN AND JERRY A. SHIELDS \\ From the Oncology Service, Wills Eye Hospital and Research Institute, Thomas Jefferson University, \\ Philadelphia, USA
}

SUMmary The clinical and histopathological findings in the eyes of 2 patients with diffuse malignant melanoma of the choroid are described. The tumours were minimally elevated and nonpigmented in the peripapillary area, and both had an associated nonrhegmatogenous retinal detachment. Extension of the tumour into the region of the lamina choroidalis in each eye appeared to cause a localised constriction of the optic nerve and subsequent swelling of the optic nerve head. In addition tumour infiltration of the nerve head was present in one eye. Ophthalmoscopic, fluorescein angiographic, and ultrasonographic characteristics that differentiate between extensive tumour infiltration of the optic nerve head and swelling secondary to other causes are presented. The importance of appreciating this difference clinically is discussed.

Diffuse choroidal melanomas are relatively rare, comprising less than $5 \%$ of all malignant melanomas of the choroid. ${ }^{2}$ The clinical diagnosis of diffuse choroidal melanoma is often difficult, and in a series reported by Font and associates ${ }^{1}$ the correct diagnosis was made preoperatively in only $36 \%$ of cases. The diagnostic difficulty results from the fact that such tumours are often flat and relatively nonpigmented, lacking the ophthalmoscopic features of most choroidal melanomas.

\section{Case reports}

CASE 1

A 69-year-old white woman presented in December 1978 with a history of recent onset of light flashes and decreased vision in the right eye. Ocular examination disclosed a best corrected visual acuity of $6 / 30$ in the right eye and 6/12 in the left eye. An afferent pupillary defect was present in the right eye. The slit-lamp examination showed normal appearances in each eye and no anterior vitreous cells were noted. Applanation intraocular pressures were $12 \mathrm{mmHg}$ in each eye.

The fundus examination of the right eye showed a swollen optic nerve head with indistinct margins. The retinal blood vessels appeared normal in calibre and configuration. Located immediately inferotemporal

Correspondence to Jerry A. Shields, MD. Oncology Service. Wills Eye Hospital, 9th and Walnut Streets, Philadelphia, PA 19107. USA. to the optic nerve head was a minimally thickened subretinal yellow mass that extended inferiorly, where it became more pigmented and attained a maximum height of about $3 \mathrm{~mm}$. Numerous small orange-brown spots, which appeared to be at the level of the retinal pigment epithelium, were present in the macular area. A shallow nonrhematogenous retinal detachment with shifting subretinal fluid was present over the lesion in the inferior peripheral fundus. The fundus of the left eye was normal. The clinical diagnosis at this time was malignant melanoma of the choroid of the right eye. An incisional ${ }^{32} \mathrm{P}$ test was negative, with $25 \%$ greater uptake over the lesion than in the control area.

The patient was observed without treatment until October 1979, when the visual acuity had decreased to counting fingers in the right eye. The nonrhegmatogenous retinal detachment had extended to involve the temporal retina also (Fig. 1), and the optic nerve head was more swollen than it had been 10 months previously.

Intravenous fluorescein angiography (Fig. 2) showed early diffuse hyperfluorescence of the optic disc and mottled areas of hyperfluorescence and hypofluorescence in the peripapillary region. The many small hypofluorescent spots located on the surface of the lesion corresponded to the orange-brown pigment seen clinically.

Contact B-scan ultrasonography of the right eye disclosed an initial echo followed by low internal 


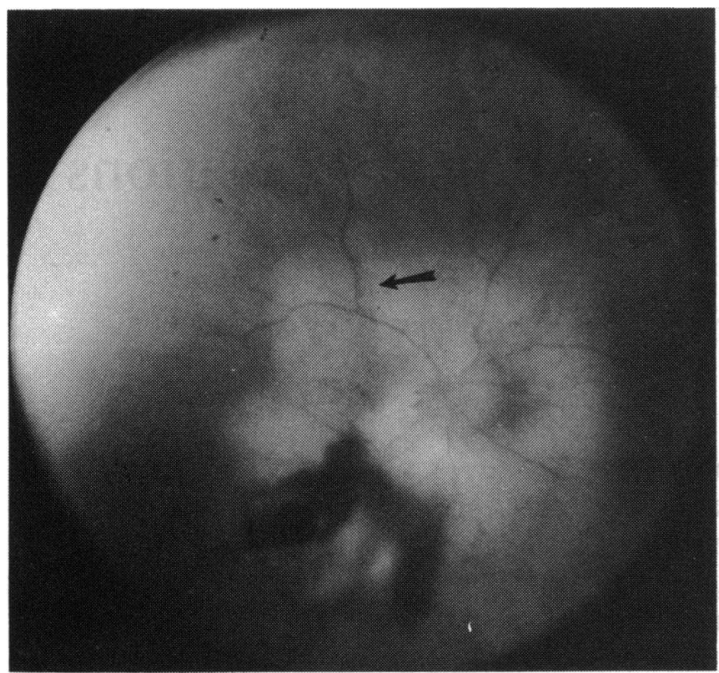

Fig. 1 Case 1. Equator-plus photograph showing the fundus appearance in October 1979. A predominantly amelanotic choroidal lesion surrounds the optic nerve head and is pigmented inferotemporally. The lesion was diffusely raised to about 1.0 to $1.5 \mathrm{~mm}$, except in the region of the inferotemporal, disc-sized, darkly pigmented nodule, where it measured approximately $3.0 \mathrm{~mm}$ in height. The associated nonrhegmatogenous retinal detachment can be seen temporally (arrow).

reflectivity in the area of the optic nerve head. The A-scan ultrasonogram showed a high initial spike followed by a progressive decrease in echo activity in the area of greatest tumour elevation inferotemporal to the nerve head.

Because of the decreasing visual acuity, increase in subretinal fluid, and increased swelling of the optic nerve head, another cutting ${ }^{32} \mathrm{P}$ test was performed. Uptake over the pigmented region of the tumour was positive at $92 \%$ greater than the opposite control quadrant. The eye was subsequently enucleated.

\section{Pathology}

Gross examination of the sectioned globe revealed a diffuse tumour, with nonpigmented and pigmented regions, measuring approximately $18 \times 18 \times 1 \mathrm{~mm}$. No evidence of pigmentation was seen on the external sclera or within the vortex veins. Low-power microscopic examination (Figs. 3, 4) disclosed a diffuse tumour replacing the choroid posteriorly. The tumour extended into the region of the optic nerve head and appeared to compress the lamina choroidalis region of the nerve. No direct invasion of the optic nerve head itself was noted. While occasional nests of tumour cells were seen in the vicinity of the meninges, no frank extension into the

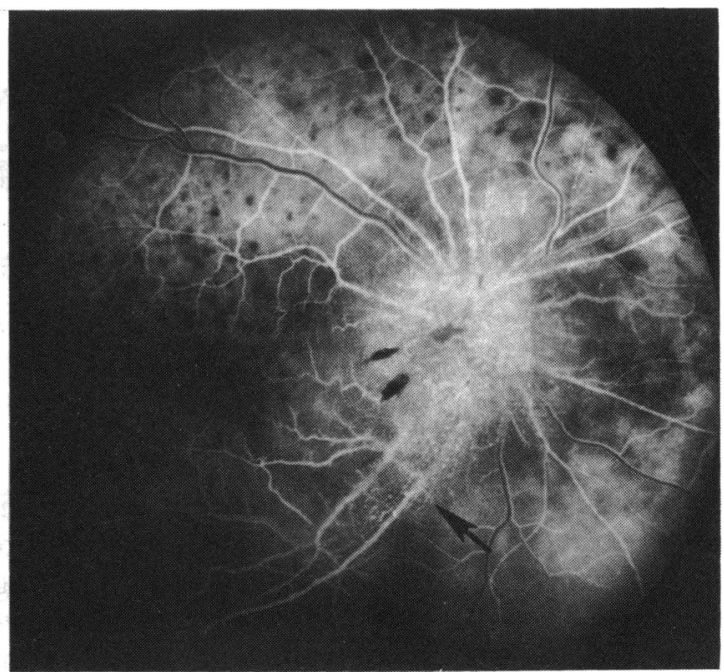

Fig. 2 Case 1. Laminar venous filling phase $45^{\circ}$ fluorescein angiogram of the right eye in October 1979. Manv small-vessel telangiectatic changes on the optic nerve head are apparent and these can be seen extending inferotemporallv from the optic nerve in the region of distribution of the radial peripapillary arteries (arrow). The optic nerve head was essentially uniformly hyperfluorescent throughout the angiogram. Mottled choroidal hyperfluorescence and hypofluorescence can be seen throughout the field. The 2 hypofluorescent foci located on the temporal portion of the optic nerve head correlate with the haemorrhages seen clinically (Fig. 1), and the many hypofluorescent spots located temporally and superiorly correspond to the orange-brown pigment present on the surface of the tumour.

subarachnoid space could be seen. The optic nerve head was diffusely thickened, and serous subretinal fluid was present inferiorly.

The tumour cells were essentially nonpigmented except in the inferior aspect of the lesion, where pigmentation had been noted ophthalmoscopically. The tumour was composed of plump epithelioid cells with prominent nucleoli and abundant cytoplasm and small areas of spindle B cells. The final diagnosis was diffuse choroidal malignant melanoma of the mixed cell type.

The patient was alive and without evidence of metastatic disease 14 months after enucleation.

\section{CASE 2}

A 66-year-old white male was first seen in December 1973 , at which time he related a history of painless, progressive visual loss in the left eye over a 4-month period. The patient had otherwise been in good health but did have a 40 -year history of cigarette smoking. 


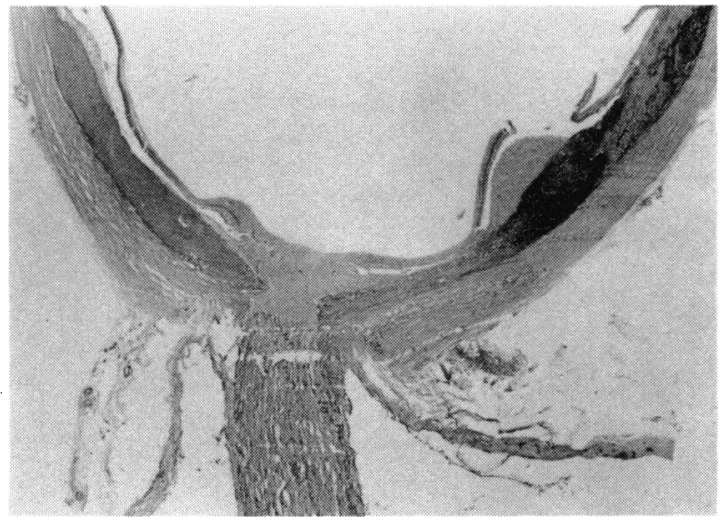

Fig. 3 Case 1. Posterior aspect of the right globe, showing diffuse infiltration of the tumour throughout the choroid. (Haematoxylin-eosin, original magnification $\times 1 \cdot 5$ ).

Ophthalmological examination disclosed a visual acuity of $6 / 12$ in the right eye and hand motions in the left eye. The applanation tonometry reading in the left eye was $14 \mathrm{mmHg}$ and in the right was $12 \mathrm{mmHg}$. The slit-lamp examination was normal in each eye and funduscopic examination of the right eye was unremarkable. Ophthalmoscopy of the left fundus revealed a minimally raised, hypopigmented, horseshoe-shaped choroidal mass occupying the posterior pole, and extending temporally and inferiorly. The mass surrounded the swollen optic nerve head (Fig. 5) and involved the total macular area. The nerve head itself was pink superiorly and had a fluffy yellow appearance inferiorly. Shifting subretinal fluid could be seen in all quadrants of the fundus.

Fluorescein angiography of the left eye (Fig. 6) showed early hyperfluorescence of the superior part of the nerve head due to filling of the many dilated surface vessels. The inferior half, that characterised by the fluffy yellow infiltrate, was relatively hypofluorescent during the early phases of the angiogram. Later in the angiogram the total nerve head became hyperfluorescent except in areas where overlying blood was present.

Contact B-scan ultrasonography disclosed the presence of diffuse choroidal thickening, subretinal fluid, and a swollen optic nerve head with moderate to high internal reflectivity.

A thorough systemic examination revealed no other abnormalities, and the patient was thought to have either metastatic carcinoma to the choroid or a diffuse choroidal melanoma. An incisional ${ }^{32} \mathrm{P}$ test was performed and was positive, with the lesion showing $191 \%$ greater uptake than the opposite uninvolved control quadrant. The eye was enucleated.

\section{Pathology}

Gross sections of the globe revealed diffuse thickening of the choroid, to a maximum of about $1.5 \mathrm{~mm}$, by a minimally pigmented tumour (Fig. 7). The retina was totally detached. Examination of the optic nerve (Fig. 8) disclosed diffuse infiltration by tumour cells extending posterior to the lamina scleralis, but not to the line of surgical excision. A focal dense accumula-

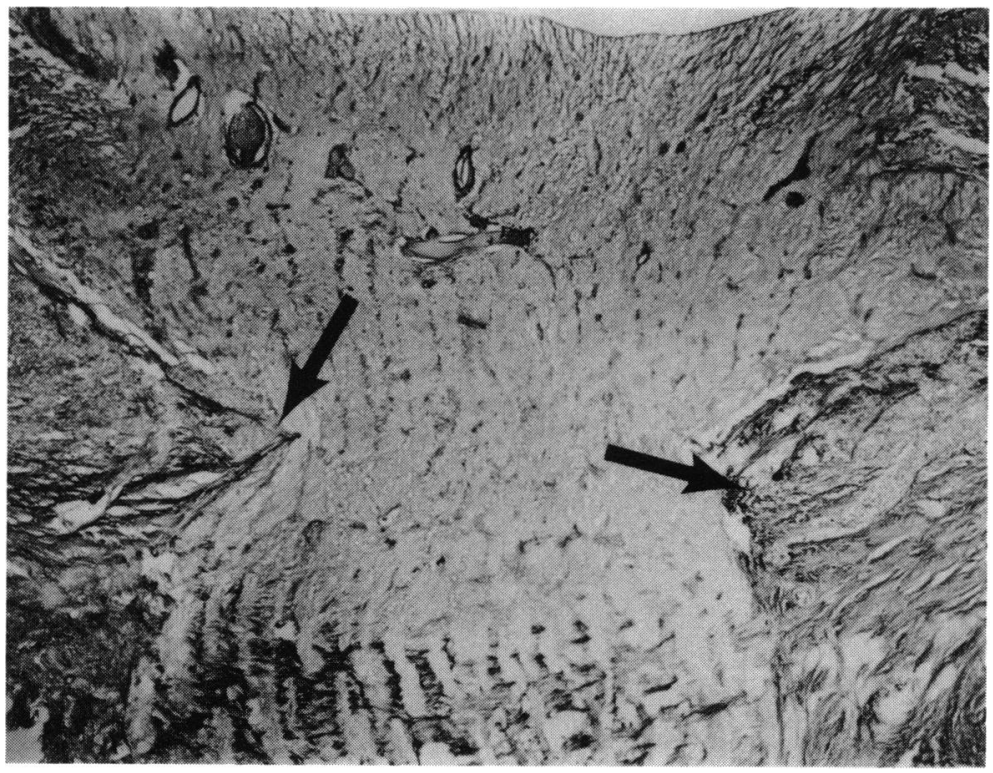

Fig. 4 Case 1. Low-power photomicrograph of the optic nerve in the right eye. The optic nerve head is swollen but not directly invaded by the choroidal tumour. Tumour can be seen compressing both sides of the optic nerve in the area of the lamina choroidalis. Occasional nests of tumour cells were seen in the region of the meninges, but no direct extension of the tumour into the subarachnoid space was observed. The furthest advance of the melanoma toward the optic nerve is indicated by the arrows. (Haematoxylin-eosin. $\times 6 \cdot 7)$. 


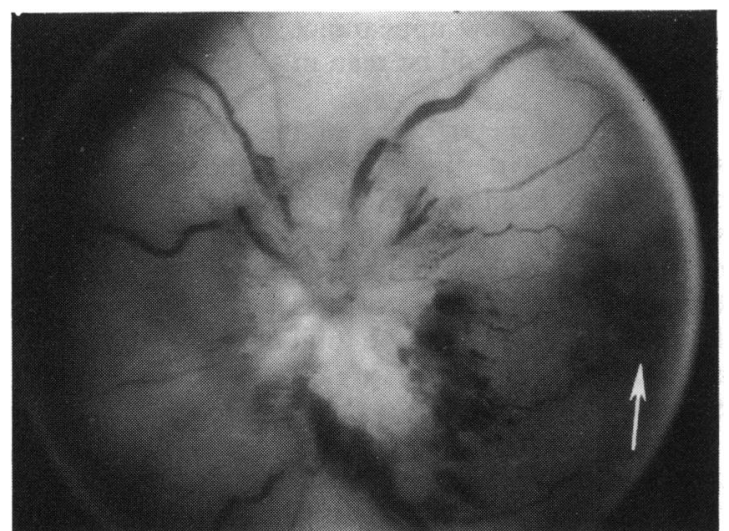

Fig. 5 Case 2. Left optic nerve head at the time of presentation. The disc is markedly swollen and the inferior half is characterised by a fluffy yellow appearance, while dilated telangiectatic vessels are more readily apparent over the superior half. Blood can be seen along the temporal and inferior borders of the nerve head, and the major retinal veins are dilated and tortuous. The nerve head is completely surrounded by hypopigmented choroidal tumour, though a small amount of pigment (arrow) can be seen in the central macular area.

tion of these cells was present centrally on the surface of the inferior half of the disc, and this inferior concentration of cells corresponded to the yellow infiltrate seen clinically on the nerve head (Fig. 5). A few scattered cytoid bodies were seen in the peripapillary region, but none were noted overlying the optic nerve head.

The choroid was replaced in the posterior pole by tumour tissue, and compression of the optic nerve by this tissue was evident in the region of the lamina choroidalis. Emissary channels in the sclera as well as periocular soft tissues nasal to the optic nerve were also involved by tumour. Spindle shaped cells with round to oval nuclei and prominent nucleoli were present in addition to areas of epithelioid cells. The histological diagnosis was choroidal melanoma of the mixed cell variety.

The patient died 6 months after enucleation, with diffuse metastatic tumour to the lungs.

\section{Discussion}

Font $e$ al. ${ }^{1}$ have described criteria for the classification of a choroidal melanoma as a diffuse type. Primary choroidal melanomas involving a minimum of one-quarter of the uvea, excluding the iris and ciliary body, and less than $7 \mathrm{~mm}$ in height are generally included in this group. The tumours described in this paper are actually less elevated than

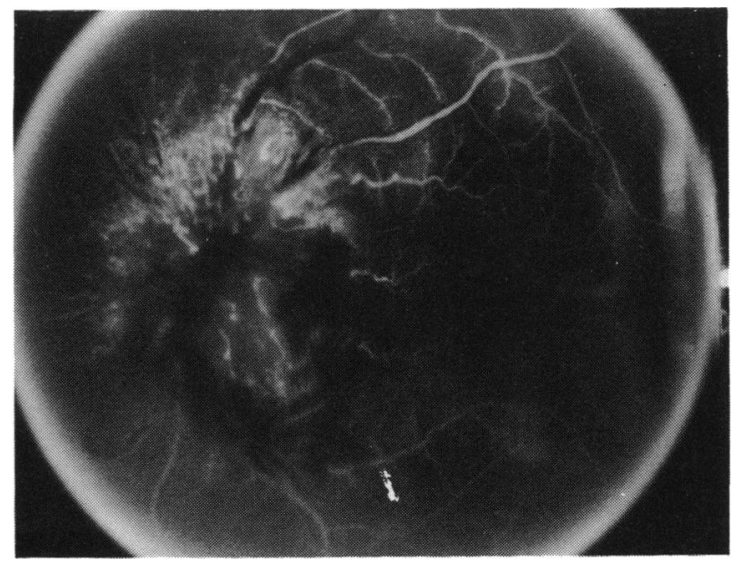

Fig. 6 Case 2. Fluorescein angiogram of the left fundus in the retinal laminar venous filling phase. The superior half of the nerve head is hvperfluorescent secondary to filling of the many dilated superficial vessels. In comparison the inferior half of the nerve head is hypofluorescent. The markedly hvpofluorescent inferior disc borders correspond to areas of superficial haemorrhage (Fig. 5), while the relatively hypofluorescent central inferior disc corresponds to the fluffv vellow infiltrate. Some blood vessels can he seen within this region of vellow tissue.

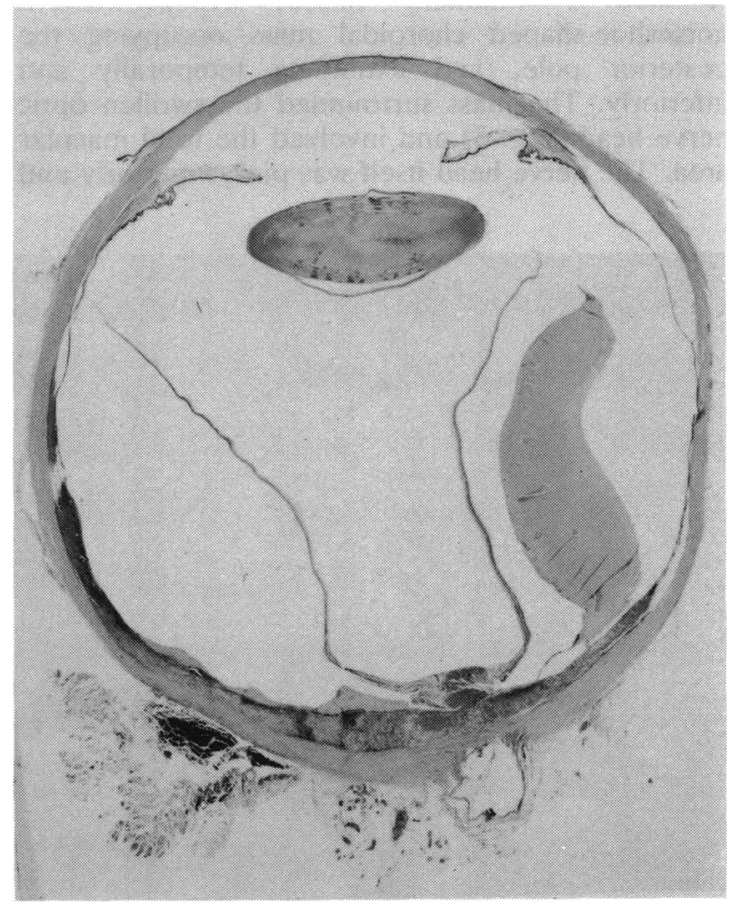

Fig. 7 Case 2. Gross section of the teft globe. The choroid is diffusely infiltrated by minimally pigmented tissue and a serous retinal detachment is evident. 


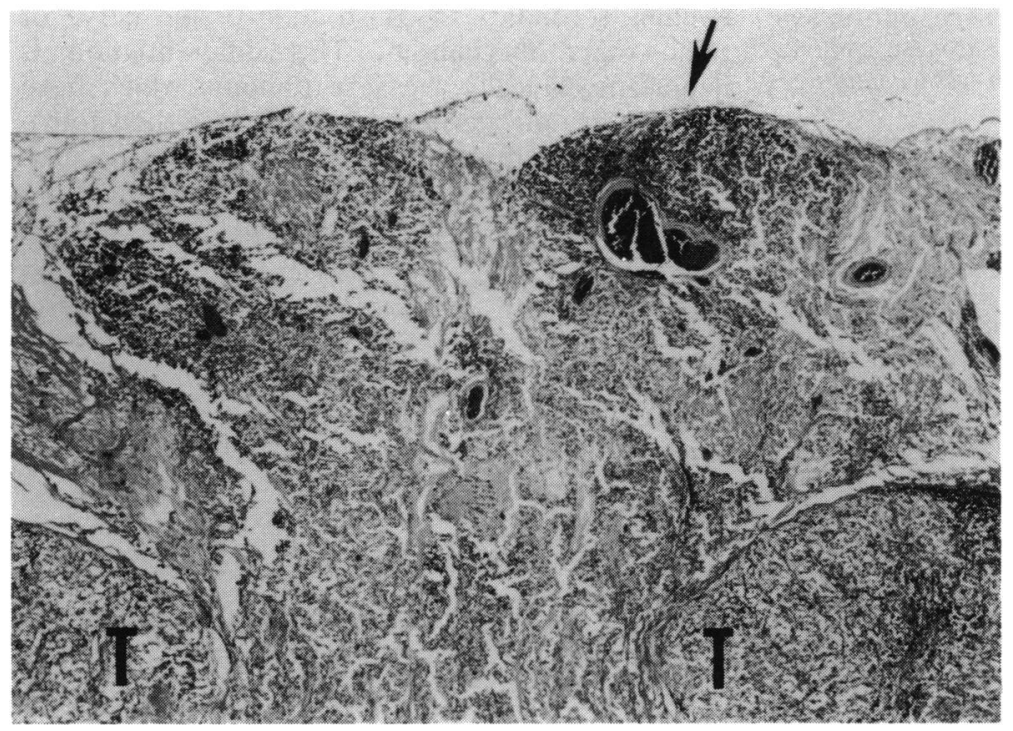

Fig. 8 Case 2. Low-power microscopic section of the left optic nerve head. Solid tumour $(T)$ can be seen compressing the nerve from both sides in the vicinitv of the lamina choroidalis, and the nerve is diffuselv infitrated bv tumour cells. A dense accumulation of tumour cells (arrow) can be seen centrallv on the surface of the nerve and corresponds to the fluffy vellow material seen clinicallv (Fig. 5). Blood is present on both sides of this superficial infiltrate, and no cvtoid bodies were observed overlving the nerve head.

many in the above authors' series, for their tumours averaged 3 to $5 \mathrm{~mm}$ in thickness.

In contrast to typical choroidal melanomas the clinical diagnosis of diffuse choroidal melanomas is often extremely difficult. From our recent experience and previous reports ${ }^{2-4}$ it seems that those entities most likely to be confused with diffuse choroidal melanomas include disciform macular degeneration, metastatic carcinoma to the choroid, choroidal haemangioma, and retinal detachment. Font et al.' found that the preoperative clinical diagnosis was correct in only $36 \%$ of 53 cases, though in another $24 \%$ some unspecified introacular tumour was suspected. In the remaining cases $(40 \%)$ the diagnosis of an intraocular tumour was unsuspected even at the time of enucleation. The difficulty of diagnosis in the past is attested to by the fact that in many eyes symptoms were present for years prior to enucleation. ${ }^{15}$ The presence of variegated pigmented regions on a raised mass, brown and orange pigment overlying the lesion, and typical ultrasonographic characteristics should arouse suspicion that a diffuse choroidal melanoma may be present. Fluorescein angiography may be helpful by showing areas of mottled hyperfluorescence and hypofluorescence, ${ }^{6}$ the latter often corresponding to clumps of macrophages containing lipofuscin pigment, ${ }^{7}$ as was the case with our first patient.

The cell type of diffuse choroidal melanomas is variable, though in the largest series described ${ }^{1} 85 \%$ were classified as either mixed cell type, pure epithelioid cell type, or necrotic. When eyes with all types of choroidal melanomas and optic nerve head extension are examined, mixed and epithelioid cell types again constitute nearly $85 \%$ of cases. ${ }^{8}$

The 5-year mortality rate following enucleation for diffuse choroidal melanomas is about $73 \% .^{1}$ The frequent delay in diagnosis, the propensity of these tumours to be exceptionally malignant, and their high incidence of extrascleral extension all account for the high death rate. These tumours may leave the globe locally via the ciliary vessels, ciliary nerves, vortex veins, and leptomeningeal sheaths. In addition orbital extension of any choroidal melanoma which has invaded the optic nerve carries a very poor prognosis for survival, ${ }^{89}$ as exemplified by the second case.

The optic nerve head swelling in our cases appeared to result from more than one mechanism. A focal constriction of the nerve by surrounding tumour in the area of the lamina choroidalis was present in each case. Possibly a disturbance in axoplasmic transport, with subsequent blockage in the area of the optic nerve head, as has been postulated to occur with papilloedema, ${ }^{10}$ may have occurred in these eyes. Hypoxia of the nerve may also have been present secondary to infiltration of the adjacent choroid and impairment of the blood supply to the nerve, and perhaps some angiogenic substance secreted by the tumour might have played a role in producing the dilated, telangiectatic nerve head vessels and swelling.

Histopathologically the second case differed substantially from the first by having invasion of the optic nerve head itself by tumour. The microscopic area of most dense tumour infiltration (Fig. 8) could be correlated ophthalmoscopically with the inferior fluffy yellow region of the swollen nerve head (Fig. 5), 
Table 1 Differentiating features between optic disc swelling without tumour invasion of the optic nerve head and swelling with tumour invasion of the nerve head in eves with diffuse choroidal melanoma

\begin{tabular}{lll}
\hline & No invasion (case I) & Invasion (case 2) \\
\hline $\begin{array}{l}\text { Ophthalmoscopic } \\
\text { appearance of swollen } \\
\text { nerve head }\end{array}$ & Diffusely pink & $\begin{array}{l}\text { Fluffy vellow } \\
\text { tissue present }\end{array}$ \\
$\begin{array}{l}\text { Fluorescein angiography } \\
\text { of nerve head } \\
\text { Early }\end{array}$ & Hyperfluorescence & $\begin{array}{l}\text { Hypofluorescence } \\
\text { (corresponding to } \\
\text { vellow tissue) }\end{array}$ \\
$\begin{array}{ll}\text { Lyte } \\
\text { Ultrasonogrfluorescence } \\
\text { head }\end{array}$ & $\begin{array}{l}\text { Hypherfluorescence } \\
\text { refinternal }\end{array}$ \\
\hline
\end{tabular}

which initially was relatively hypofluorescent with fluorescein angiography (Fig. 6). While this area appeared clinically similar to what is seen with multiple infarctions of the nerve fibre layer, only a few scattered cytoid bodies were seen microscopically in the peripapillary region and none were observed over the nerve head. In the first case the optic nerve head, which was swollen but not infiltrated by tumour, was congruously pink and uniformly hyperfluorescent during the early phases of the angiogram. Ultrasonographically it was noted that the optic nerve head without tumour infiltration showed low internal reflectivity, while that with infiltration showed moderate to high reflectivity. A summary of the differentiating features that help to determine whether tumour invasion of the optic nerve head is present is shown in Table 1.

Thus it appears that ophthalmoscopically, fluorescein angiographically, and ultrasonographically it is possible to differentiate direct tumour invasion of the optic nerve head from nerve head swelling secondary to constriction of the nerve or some other mechanism. This differentiation is important clinically, because tumours which have invaded the optic nerve head are likely to show retrolaminar invasion of the nerve as well, thus necessitating special attempts to obtain a long section of the optic nerve at the time of enucleation. ${ }^{8}$

This study was supported in part by the Retina Research and Development Foundation, Philadelphia, the Ocular Oncology Fund. Wills Eve Hospital, and the Pennsvlvania Lions Sight Conservation and Eye Research Foundation. Inc.

The first patient was referred by Drs Morton Seelenfreund and Dennis Freilich and the second was referred bv $\mathrm{Dr}$ William $\mathrm{H}$.

Annesley Jr.

\section{References}

1 Font RL. Spaulding RG, Zimmerman LE. Diffuse malignant melanoma of the uveal tract: a clinicopathologic report of 54 cases. Trans Am Acad Ophthalmol Otolarvngol 1968: 72: 877-95.

2 Reese AB. Howard GM. Flat uveal melanomas. Am J Ophthalmol 1967: 64: 1021-8.

3 Ferry AP. Lesions mistaken for malignant melanoma of the posterior uvea: a clinicopathologic analysis of 100 cases with ophthalmoscopically visible lesions. Arch Ophthalmol 1964: 72: $463-9$.

4 Shields JA. Zimmerman LE. Lesions simulating malignant melanoma of the posterior uvea. Arch Ophthalmol 1973: 89: $466-71$.

5 Parson JH. Diffuse sarcomata of the uveal tract. Arch Ophthalmol 1904: 33: 101-12.

6 Edwards WC. Layden WE. MacDonald R. Fluorescein angiographv of malignant melanoma of the choroid. Am J Ophthalmol 1969: 68: 797-808

7 Shields JA. Rodrigues MM. Sarin LK. Tasman WS. Anneslev WH. Jr. Lipofucsin pigment over benign and malignant choroidal melanomas. Trans Am Acad Ophthalmol Otolaryngol 1975: 81: $871-81$.

8 Shammas HF. Blodi FC. Peripapillary choroidal melanomas. Arch Ophthalmol 1978: 96: 440)-5.

9 Spencer WH. Optic nerve extension of intraocular neoplasms. Am J Ophthalmol 1975: 80: 465-71.

10 Tso MOM. Havreh SS. Optic disc edema in raised intracranial pressure. IV. Axoplasmic transport in experimental papilledema. Arch Ophthalmol 1977: 95: 1458-62. 